American Journal of Immunology, 1 (1): 6-11, 2005

ISSN 1553-619X

(C) 2005 Science Publications

\title{
Extracts of Pumpkin (Cucurbita pepo L.) Seeds Suppress Stimulated Peripheral Blood Mononuclear Cells in vitro
}

\author{
${ }^{1,2}$ Christiana Winkler, ${ }^{1}$ Barbara Wirleitner, ${ }^{1}$ Katharaina Schroecksnadel \\ ${ }^{3}$ Harald Schennach and ${ }^{1,2}$ Dietmar Fuchs \\ ${ }^{1}$ Division of Biological Chemistry, Biocentre, Innsbruck Medical University \\ ${ }^{2}$ Ludwig Boltzmann Institute of AIDS-Research, Innsbruck, Austria \\ ${ }^{3}$ Central Institute of Blood Transfusion and Immunology, University Hospital
}

\begin{abstract}
In the traditional medicine in North America and Mexico, pumpkin seeds have been used as an anthelmintic agent and for supportive treatment in functional disorders of the bladder. Also antiinflammatory and cardioprotective activity of pumpkin seeds is discussed. Three different extracts of pumpkin seeds were prepared and effects were investigated in unstimulated human peripheral blood mononuclear cells and in cells stimulated with the mitogens phytohaemagglutinin and concanavalin A in vitro. Tryptophan degradation and neopterin concentrations were measured in the supernatants allowing to detect biochemical changes induced by cytokine interferon- $\gamma$. Extracts of pumpkin seeds suppressed mitogen-induced neopterin production and tryptophan degradation in a dose-dependent way. Data demonstrate capacity of pumpkin extracts to modulate immunobiochemical pathways induced by interferon- $\gamma$. Findings imply an immunoregulatory potential of compounds contained in pumpkin seeds.
\end{abstract}

Key words Cucurbita pepo L., pumpkin, neopterin, tryptophan, immune modulation

\section{INTRODUCTION}

Cucurbita (C.) pepo (variety convar. citrullinina GREB. var. styriaca GREB), a pumpkin used in medical applications, is an annual plant with yellow flowers. It has a climbing stem up to $12 \mathrm{~m}$ long and a fruit with a round shape and fibrous flesh and is cultivated mainly in Austria, Slowenia, Hungaria and Mexico. The high quality edible oil is used for salad dressing. Fruits consist of up to $50 \%$ fatty oil, carotenoids, proteins, tocopherols, phytosterols and phytoestrogens as well ${ }^{[1-4]}$. Pumpkin seed has been used in traditional medicine in North America and Mexico since long as an antihelmintic agent and for supportive treatment in functional disorders of the bladder and for difficulties in urination ${ }^{[5]}$. Childhood enuresis nocturna and irritable bladder have been treated successfully with pumpkin seed ${ }^{[6]}$ it has also been used to eradicate tapeworm ${ }^{[7]}$. Its modern clinical use is comparable to its traditional applications in Northern American aboriginal medicine. Pumpkin seeds are considered an alternative treatment for stage I and II benign prostatic hyperplasia and for irritable bladder ${ }^{[8]}$. Antiinflammatory and cardioprotective effects of Cucurbita may relate to an influence of plant compounds on immunocompetent cells.

Tryptophan degradation and neopterin formation are induced during Th1-type immune response ${ }^{[9]}$. Both these biochemical pathways are stimulated by cytokine interferon- $\gamma$ (IFN- $\gamma$ ): in macrophages IFN- $\gamma$ induces GTP-cyclohydrolase I, the key enzyme for neopterin formation $^{[10]}$. In parallel, IFN- $\gamma$ induces enzyme indoleamine (2,3)-dioxygenase (IDO) which initiates the conversion of tryptophan via the kynurenine pathway $^{[11]}$. IDO activity can be estimated by calculating the ratio of the product kynurenine and the substrate tryptophan $(\mathrm{kyn} / \mathrm{trp})^{[12]}$. The measurement of neopterin and of kyn/trp can be applied to detect immune activation in patients ${ }^{[10-12]}$ and is useful also to monitor endogenous production of interferon- $\gamma$ in vitro $^{[9]}$. Using peripheral blood mononuclear cells (PBMC) stimulated with mitogens, a potential immunomodulatory property of compounds or pharmaceutics can be easily and sensitively monitored $^{[13,14]}$ recently, suppressive effects of plant extracts like Uncaria tomentosa ${ }^{[15]}$ or Hypericum perforatum $^{[16]}$ were observed. In this study, we investigated the influence of three pumpkin seed preparations on stimulated and unstimulated peripheral blood mononuclear cells (PBMC) of healthy donors.

\section{MATERIALS AND METHODS}

Preparation of seeds extracts: Pumpkin seeds from biological cultivation (Kürbiskerne, Engelbert Perlinger Bioprodukte, Austria) were applied for preparing the

Corresponding Author: Dietmar Fuchs, Division of Biological Chemistry, Biocentre, University of Innsbruck, Fritz Pregl Strasse 3, A-6020 Innsbruck, Austria, Phone: +43 512507 3519, Telefax: +43 5125072865 
cold and the hot extracts used for experiments with PBMC. Seeds were finely grounded and $10 \mathrm{~g}$ of the powder were added to $100 \mathrm{ml}$ of supplemented RPMI. The cold extract was shaken for 10 minutes, the hot extract was boiled for 1 minute. The calculated highest final concentration in the experiments was $50 \mu \mathrm{g} \mathrm{mL}^{-1}$ seeds for the cold and hot extracts.

A third extract was prepared from pumpkin capsules (Fa. Magister Doskar, Vienna, Austria) purchased at the drugstore: 1 capsule $(0.72 \mathrm{~g})$ was added to $30 \mathrm{ml}$ supplemented RPMI and mixed, the highest concentration reached was $38.4 \mu \mathrm{g} \mathrm{mL}^{-1}$ seeds from capsule. All extracts were sterile filtered at 0.2 $\mu \mathrm{m}$, thereby non-dissolved material was removed frozen at $-20^{\circ} \mathrm{C}$ until used.

Isolation and stimulation of human PBMC: PBMC were isolated from whole blood obtained from healthy donors. Separation of blood cells was performed using density centrifugation (Lymphoprep, Nycomed Pharma AS, Oslo, Norway). After isolation, PBMC were washed three times in phosphate buffered saline containing $0.2 \% 0.5 \mathrm{mM}$ EDTA. Cells were maintained in RPMI 1640 supplemented with $10 \%$ heat-inactivated fetal calf serum (Biochrom, Berlin, Germany), $1 \%$ of $200 \mathrm{mM}$ glutamin (Serva, Heidelberg, Germany) and $0.1 \%$ of gentamycine $\left(50 \mathrm{mg} \mathrm{mL}{ }^{-1}\right.$, Bio-Whittaker, Walkersville, MD) in a humidified atmosphere containing $5 \% \mathrm{CO}_{2}$ for $48 \mathrm{~h}$. For each experiment, a fresh preparation of PBMC from four different healthy blood donors was used. Isolated PBMCs were plated at a density of $1.5 \times 10^{6}$ cells $\mathrm{mL}^{-1}$ in supplemented RPMI 1640, preincubated for 30 minutes with or without pumpkin seed extracts and stimulated or not with mitogens phytohaemagglutinin (PHA, Sigma, Vienna, Austria) and concanavalin A (Con A, Sigma, Vienna, Austria) for $48 \mathrm{~h}$.

Measurements: After incubation, supernatants were harvested and neopterin concentrations were determined by ELISA (BRAHMS Diagnostica, Berlin, Germany) according to the manufacturers instructions with a detection limit of $2 \mathrm{nM}$. Tryptophan and kynurenine concentrations were quantified by high pressure liquid chromatography (HPLC) using 3-nitroL-tyrosine as internal standard ${ }^{[17]}$. Kyn/trp was calculated and expressed as $\mu \mathrm{mol}$ kynurenine $\mathrm{mmol}^{-1}$ tryptophan.

Statistics: For each stimulation, at least four experiments using blood from different donors were performed with two parallels each. Data are presented as mean \pm S.E.M. To correct for moderate interindividual differences, results are presented as fold of unstimulated control of each experiment. For comparison of grouped data, Mann-Whitney U-test was applied. P-values below 0.05 were considered to indicate significant differences.

\section{RESULTS}

Unstimulated cells: After an incubation period of 48h, average neopterin concentration of $5.8 \mathrm{nM} \pm 1.7$ was detected in supernatants of unstimulated cells. Average concentrations of tryptophan and kynurenine were 23.3 $\pm 3.9 \mu \mathrm{M}$ and $2.0 \pm 0.5 \mu \mathrm{M}$ respectively, kyn/trp was $102.3 \pm 44.9 \mu \mathrm{mol} \mathrm{mol}^{-1}$.

Mitogen-stimulated cells: Stimulation of cells with 10 $\mu \mathrm{g} \mathrm{mL}{ }^{-1}$ of mitogens PHA or Con A increased neopterin production $16.8 \pm 6.7 \mathrm{nM}(\mathrm{P}<0.01)$. When the extracts where added, neopterin concentrations declined dose-dependently (Fig. 1). With $1-50 \mu \mathrm{g} \mathrm{mL}^{-1}$ of the cold and the hot extracts or $8 \mu \mathrm{g} \mathrm{mL} L^{-1}$ of the capsules respectively, neopterin concentrations were significantly suppressed. At the highest concentration neopterin levels reached baseline of unstimulated cells (all $\mathrm{P}<0.01$ compared to the control).

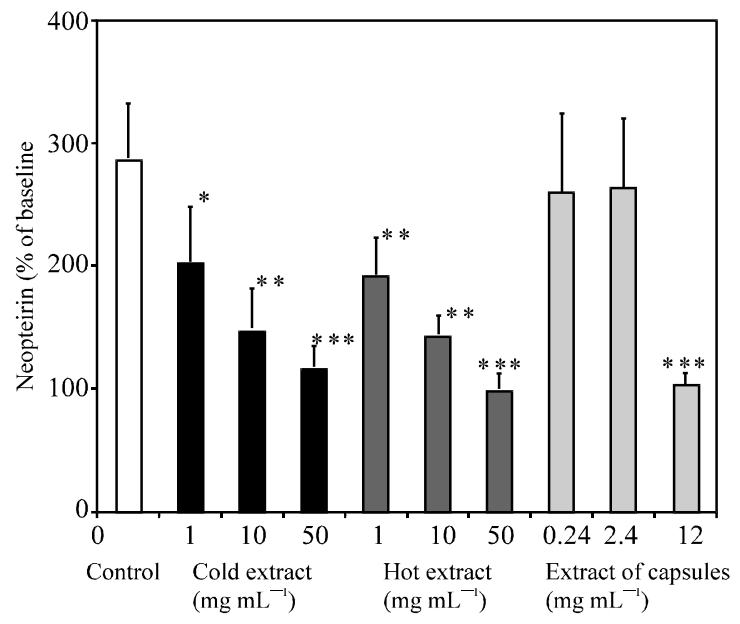

Fig. 1: Neopterin concentration in peripheral blood mononuclear cells stimulated with $10 \mu \mathrm{g} \mathrm{mL}^{-1}$ phytohaemagglutinin (PHA) and $10 \mu \mathrm{gL}^{-1}$ concanavalin A (Con A) (white bars) and coincubated with a cold extract (black bars), a hot extract (hatched bars) or an extract of capsules (grey bars) of pumpkin seeds for 48 hours (*P $<0.05, * * \mathrm{P}<0.01, * * * \mathrm{P}<0.001$ compared to the control; data are presented as fold-of-control = unstimulated cells)

Tryptophan degradation as indicated by kyn/trp, was increased in stimulated PBMC as compared with unstimulated cells (Fig. 2) kyn/trp was 441-fold ( \pm 2.9 ) higher than in unstimulated controls. All extracts inhibited stimulation-induced tryptophan degradation and reduced kyn/trp ratio significantly (at the highest concentration all $\mathrm{P}<0.001$ compared to the control). 


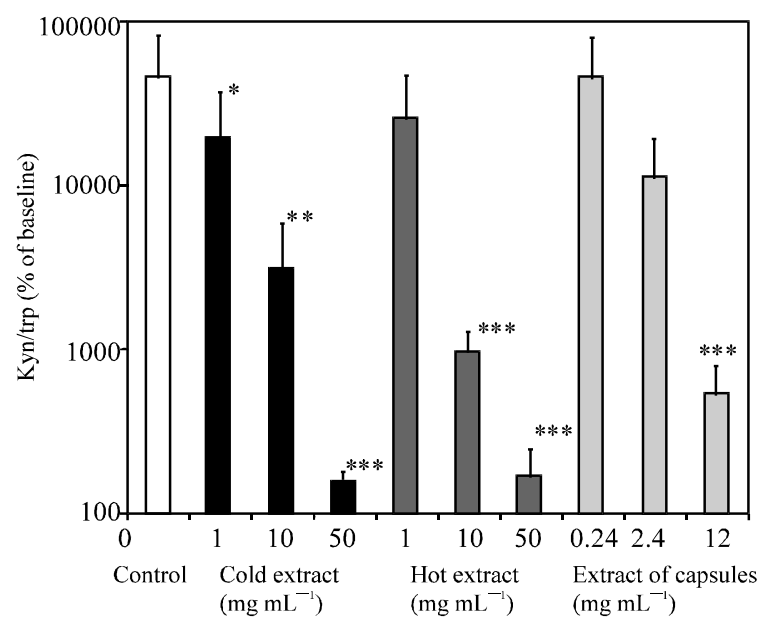

Fig. 2: Kynurenine to tryptophan ratio (kyn/trp, $\mu \mathrm{mol}$ $\mathrm{mmol}^{-1}$ ) in peripheral blood mononuclear cells stimulated with $10 \quad \mu \mathrm{g} \quad \mathrm{mL}^{-1}$ phytohaemagglutinin (PHA) and $10 \mu \mathrm{g} \mathrm{mL}^{-1}$ concanavalin A (Con A) (white bars) and coincubated with a cold extract (black bars), a hot extract (hatched bars) or an extract of capsules (grey bars) of pumpkin seeds for 48 hours $(* \mathrm{P}<0.05, * * \mathrm{P}<0.01, * * * \mathrm{P}<0.001$ compared to the control; data are presented as fold-of-control = unstimulated cells; note logscale of kyn/trp)

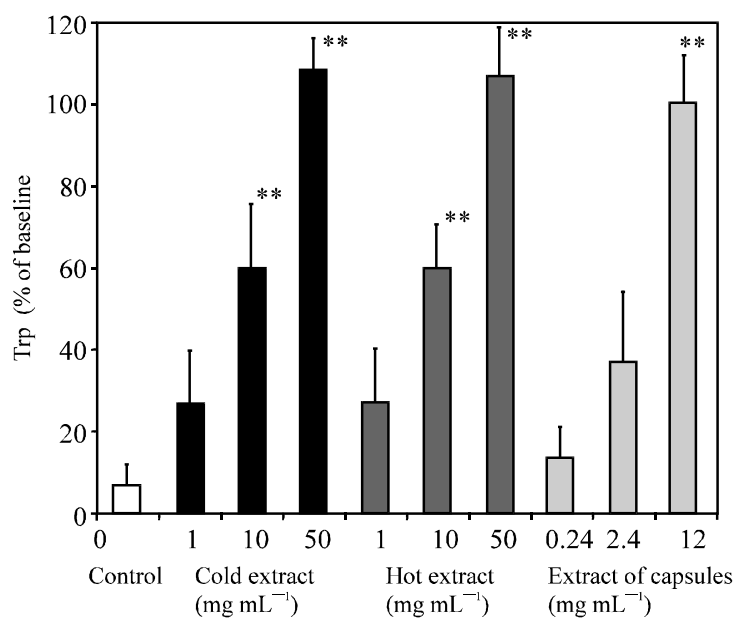

Fig. 3: Concentration of tryptophan in peripheral blood mononuclear cells stimulated with $10 \mu \mathrm{g} \mathrm{mL}^{-1}$ phytohaemagglutinin (PHA) and co-incubated with a cold extract, a hot extract or an extract of capsules of pumpkin seeds for 48 hours (*P $<0.05, * * \mathrm{P}<0.01$ compared to the control; data are presented as fold-of-control of unstimulated cells $=\mu \mathrm{mol} \mathrm{L}{ }^{-1}$ )

Tryptophan concentration were significantly decreased in supernatants of PBMC upon stimulation with mitogens (Fig. 3). Treatment of stimulated cells with pumpkin seeds extracts increased tryptophan levels at the highest dose used, tryptophan concentrations returned to the concentrations of unstimulated PBMC.

\section{DISCUSSION}

Extracts of pumpkin seeds suppressed tryptophan degradation and neopterin production, which both are increased in stimulated PBMC. At the highest concentration used, the mitogen-induced neopterin formation and tryptophan degradation were completely suppressed and neopterin, tryptophan and kynurenine concentrations reached baseline levels of unstimulated cells. The effects of test substances were dosedependent the hot and cold extracts behaved in a similar way, while the extract from the capsules appeared to be slightly less effective. The capacity of pumpkin seed extracts to suppress neopterin production and tryptophan degradation in stimulated PBMC was comparable to that obtained earlier when using, e. g., anti-inflammatory cytokines ${ }^{[9]}$, HMG-reductase inhibitor atorvastatin ${ }^{[13]}$, green and black tea ${ }^{[14]}$, plant extracts $^{[15,16]}$ and of wine ${ }^{[18]}$ in an identical experimental setting. As both these biochemical changes are triggered by the cytokine IFN- $\gamma^{[9-11]}$, data suggest a suppressive effect of the seed extracts on the formation and release of this particular cytokine in mitogen stimulated PBMCs.

Increased neopterin concentrations and accelerated tryptophan degradation have been observed in a variety of diseases including infections, autoimmune syndromes, cardiovascular disease and also cancer ${ }^{[10,11,19-23]}$. In these clinical conditions, immune activation not only parallels the course of the disease, greater deviations from normal represent an early sign of poor prognosis. Moreover, IDO activity has been described as an important immune escape mechanism in malignant tumor cells recently ${ }^{[24]}$. At least in vitro, pumpkin seeds extracts were able to interfere with immune activation and cytokine cascades. Thereby pumpkin seeds may down-regulate various biochemical pathways which are linked with an activated cellular immune system and are induced by cytokine IFN- $\gamma$, e.g. IDO or GTP-cyclohydrolase I and also the production of ROS. IFN- $\gamma$ is an important cytokine within antimicrobial host defence which enforces forwardregulatory T-cell response mechanisms: IFN- $\gamma$ is especially important during the acute phase of the immune response supporting T-cell activation ${ }^{[25]}$. As part of its antimicrobial and cytocidal activity, IFN- $\gamma$ also induces production and release of reactive oxygen species (ROS) in macrophages ${ }^{[26]}$. The increased production of oxidants and free radicals during inflammatory disorders including coronary heart disease has become widely recognised as integral component of cell and tissue injury ${ }^{[27]}$ IFN- $\gamma$ is one of the most important mediators of ROS formation ${ }^{[26]}$. 
In our experiments, all extracts (highest concentrations of extracts were $50 \mu \mathrm{g} \mathrm{mL}^{-1}$ of seeds and $8 \mu \mathrm{g} \mathrm{mL}^{-1}$ capsules) were comparably effective to suppress stimulation-induced neopterin formation and tryptophan degradation at the highest concentrations, because according to the manufacturer content of one capsule $\left(=8 \mu \mathrm{g} \mathrm{mL}^{-1}\right)$ corresponded to $10 \mu \mathrm{g} \mathrm{mL}^{-1}$ of pumpkin seeds. It is unclear which compounds of seeds are responsible for the observed effects. Inhibitory effect of seed may relate to antifungal and antibacterial compounds, from which seeds take advantage as a kind of self-protection against environmental pathogens before growth of the plant is initiated. A number of compounds of the seeds has been investigated for their cytotoxic, hepatoprotective, anti-inflammatory and cardioprotective effects ${ }^{[28]}$ and as diabroticites ${ }^{[29,30]}$. Recently glycosides with lignan and phenol belonging to the group of phytoestrogens have been discovered in pumpkin seeds. Secoisolariciresinol, a lignan-glycoside, is one of the best known representative which, depending on dosage may have estrogenic and antiestrogenic effects. Further investigations have been made to prove activity of lignans preventing hyperproliferation of prostatic cells and improving irritable bladder symptoms ${ }^{[5]}$. C. andreana exhibited potent anticancer and cyclooxygenase- 2 inhibitory activities, the purification of the extract yielded four pure anticancer, cyclooxygenase inhibitory and antioxidant compounds ${ }^{[31]}$. These different compounds from Cucurbita maxima as used in Brazilian folk medicine were not toxic for rats and swine ${ }^{[32]}$. Likewise, no toxicity was observed at the full range of concentrations applied in our study.

The inhibitory effect of pumpkin seeds on PBMC is not a specific effect for this seed. Extracts of plants have been found recently to suppress stimulationinduced neopterin production and tryptophan degradation in $\mathrm{PBMC}^{[15,16]}$, data indicated that antioxidant content of plants extracts was responsible for their immunosuppressive capacity ${ }^{[32,33]}$. Results obtained now with pumpkin seeds extracts were almost identical to the earlier data and show that also compounds in seeds possess this immunosuppressive attribute again antioxidant compounds could be involved. Antioxidants not only act as chemical antioxidants detoxifying ROS, they may also reduce the formation of ROS by suppressing effects which are triggered by IFN- $\gamma$. The extrapolation of our in vitro data to the in vivo situation is still questionable. Beneficial health effects of antioxidants in nutrients is still highly discussed in a recent epidemiological study shows that intake of green vegetables was associated with a significant reduction of cardiovascular disorders but not of other major diseases ${ }^{[34]}$.

The extracts themselves contained tryptophan: at 1 $\mu \mathrm{g} \mathrm{mL}^{-1}$ seeds the concentration was $0.7 \mu \mathrm{M}$ and thus very low, at $10 \mu \mathrm{g} \mathrm{mL}^{-1}$ it increased to $3.9 \mu \mathrm{M}$ and at
$50 \mu \mathrm{g} \mathrm{mL} L^{-1}$ it reached around $16.6 \mu \mathrm{M}$. However, tryptophan content did not influence the results of our investigations. Tryptophan content of the pure culture media used was still higher than the increase observed after addition of seeds extracts the highest concentrations of seeds not only further increased tryptophan concentrations by inhibition of its degradation, in parallel, kynurenine levels were significantly reduces. Moreover, the determination of neopterin concentrations was totally independent from tryptophan content of seeds.

In summary, our in vitro study demonstrates that extracts of Cucurbita interfere with immunologic pathways which involve Th-1 type cytokine IFN- $\gamma$. Data show that seed compounds have similar immunosuppressive activity as plant extracts and findings may relate to the health beneficial effects of pumpkin seeds extracts. The extracts obviously contain compounds which upon isolation and identification could be useful for the development of new immunosuppressants.

\section{ACKNOWLEDGMENT}

This work was supported by the Austrian Federal Ministry of Social Affairs and Generations and we thank Mrs. Astrid Haara for excellent technical assistance.

\section{REFERENCES}

1. Sicilia, T., H.B. Niemeyer, D.M. Honig and M. Metzler, 2003. Identification and stereochemical characterization of lignans in flaxseed and pumpkin seeds. J. Agric. Food Chem. 26: 51: 1181-8.

2. Rodriguez, J.B., E.G. Gros, M.H. Bertoni and P. Cattaneo, 1996. The sterols of Cucurbita moschata ("calabacita") seed oil. Lipids, 31: 1205-8.

3. Murkovic, M., A. Hillebrand, J. Winkler, E. Leitner and W. Pfannhauser, 1996. Variability of fatty acid content in pumpkin seeds (Cucurbita pepo L.). Z. Lebensm Unters Forsch, 203: 216-9.

4. Matus Z, P. Molnar and L.G. Szabo, 1993. Main carotenoids in pressed seeds (Cucurbitae semen) of oil pumpkin (Cucurbita pepo convar. pepo var. styriaca). Acta Pharm. Hung., 63: 247-56.

5. Srivastava, M.C. and S.W. Singh, 1967. Anthelmintic activity of Cucurbita maxima (kaddu) seeds. Indian J. Med. Res., 55: 629-32.

6. Weiss, R.F., 1988. Herbal Medicine. Beaconsfield, England: Beaconsfield Publishers.

7. Dreikorn, K., R. Berges, L. Pientka and U. Jonas, 2002. Phytotherapy of benign prostatic hyperplasia. Current evidence-based evaluation. Urologe A., 41: 447-51. 
8. Zdunczyk, Z., D. Minakowski, S. Frejnagel and M. Flis, 1999. Comparative study of the chemical composition and nutritional value of pumpkin seed cake, soybean meal and casein. Nahrung, 43: 392-5.

9. Weiss, G., C. Murr, H. Zoller, M. Haun, B. Widner, C. Ludescher and D. Fuchs, 1999. Modulation of neopterin formation and tryptophan degradation by Th1- and Th2-derived cytokines in human monocytic cells. Clin. Exp. Immunol., 116: 435-40.

10. Widner, B., C. Murr, B. Wirleitner, C. Mayr, N. Spöttl, G. Baier-Bitterlich and D. Fuchs, 1999. The importance of neopterin as a laboratory diagnostic marker of immune activation. Pteridines, 10: 10111.

11. Wirleitner, B., G. Neurauter, K. Schröcksnadel, B. Frick and D. Fuchs, 2003. Interferon- $\gamma$-induced conversion of tryptophan: Immunologic and neuropsychiatric aspects. Current Med. Chem., 10: 1581-91.

12. Fuchs, D., A.A. Möller, G. Reibnegger, E. Stöckle, E.R. Werner and H. Wachter, 1990. Decreased serum tryptophan in patients with HIV-1 infection correlates with increased serum neopterin and with neurologic/psychiatric symptoms. J. Acquir. Immun. Defic. Syndr., 3: 873-6.

13. Neurauter, G., B. Wirleitner, A. Laich, H. Schennach, G. Weiss and D. Fuchs, 2003. Atorvastatin suppress interferon- $\gamma$-induced neopterin formation and tryptophan degradation in human peripheral blood mononuclear cells and in monocytic cell lines. Clin. Exp. Immunol., 131: 264-7.

14. Zvetkova, E., B. Wirleitner, N.T. Tram, H. Schennach and D. Fuchs, 2001. Aqueous extracts of Crinum latifolium (L.) and Camellia sinensis show immunomodulatory properties in human peripheral blood mononuclear cells. Intl. Immunopharmacol., 1: 2143-50.

15. Winkler, C., B. Wirleitner, K. Schroecksnadel, H. Schennach, E. Mur and D. Fuchs, 2004. In vitro effects of two extracts and two pure alkaloid preparations of Uncaria tomentosa on peripheral blood mononuclear cells. Planta Med., 70: 205-10.

16. Winkler, C., B. Wirleitner, K. Schroecksnadel, H. Schennach, E. Mur and D. Fuchs, 2004. St. John's wort (Hypericum perforatum) counteracts cytokine-induced tryptophan catabolism in vitro. Biol. Chem., 385: 1197-202.

17. Widner, B., E.R.Werner, H. Schennach, H. Wachter and D. Fuchs, 1997. Simultaneous measurement of serum tryptophan and kynurenine by HPLC. Clin. Chem., 43: 2424-6.
18. Neurauter, G., B. Wirleitner, K. Schroecksnadel, H. Schennach and D. Fuchs, 2004. Wine and grape juice modulate biochemical pathways in stimulated human peripheral blood mononuclear cells. Pteridines, 15: 1-9.

19. O'Shea, J.J. and W.E. Paul, 2002. Regulation of $\mathrm{T}(\mathrm{H}) 1$ differentiation-controlling the controllers. Nat. Immunol., 3: 506-8.

20. Nathan, C.F., H.W. Murray, M.E. Wiebe and B.Y. Rubin, 1983. Identification of interferon-gamma as the lymphokine that activates human macrophage oxidative metabolism and antimicrobial activity. J. Exp. Med., 158: 670-89.

21. Gutteridge, J.M. and B. Halliwell, 2000. Free radicals and antioxidants in the year 2000. A historical look to the future. Ann.N.Y. Acad. Sci., 899: 136-47.

22. Zangerle, R., B. Widner, G. Quirchmair, G. Neurauter, M. Sarcletti and D. Fuchs, 2002. Effective antiretroviral therapy reduces degradation of tryptophan in patients with HIV-1 infection. Clin. Immunol., 104: 242-7.

23. Widner, B., N. Sepp, E. Kowald, U. Ortner, B.Wirleitner, P. Fritsch, G. Baier-Bitterlich and D. Fuchs, 2000. Enhanced tryptophan degradation in systemic lupus erythematosus. Immunobiology, 201: 621-30.

24. Wirleitner, B., R. Rudzite, G. Neurauter, C. Murr, U. Kalnins, A. Erglis, K. Trusinskis and D. Fuchs, 2003. Immune activation and degradation of tryptophan in coronary heart disease. Eur. J. Clin. Invest., 33: 550-4.

25. Zouridakis, E., P. Avanzas, R. Arroyo-Espliguero, S. Fredericks and J.C. Kaski, 2004. Markers of inflammation and rapid coronary artery disease progression in patients with stable angina pectoris. Circulation, 110: 1747-53.

26. Schroecksnadel, K., C. Winkler, L.C. Fuith and D. Fuchs, 2005. Tryptophan degradation in patients with gynecological cancer correlates with immune activation. Cancer Lett. (in press).

27. Uyttenhove, C., L. Pilotte, I. Theate, V. Stroobant, D. Colau, N. Parmentier, T. Boon, B.J. Van den Eynde, 2003. Evidence for a tumoral immune resistance mechanism based on tryptophan degradation by indoleamine 2,3-dioxygenase. Nat. Med., 9: 1269-74.

28. Miro, M., 1995. Cucurbitacins and their pharmacological effects. Phytother. Res., 9: 159-68.

29. Metcalf, R.L., R.A. Metcalf and A.M. Rhodes,1980. Cucurbitacins as kairomones for diabroticite beetles. Proc. Natl. Acad. Sci. USA., 17: 3769-72.

30. Metcalf, R.L., A.M. Rhodes, R.A. Metcalf, J.E. Ferguson, E.R. Metcalf and P. Yung, 1982. Cucurbitacin contents and diabroticites (Coleoptera; Chrysomelidae) feeding upon Cucurbita ssp. Environ. Entomol., 11:931-7. 
31. Jayaprakasam, B., N.P. Seeram and M.G. Nair, 2003. Anticancer and antiinflammatory activities of cucurbitacins from Cucurbita andreana. Cancer Lett., 189:11-6.

32. De Queiroz-Neto, A., M.I. Mataqueiro, A.E. Santana and A.C. Alessi, 1994. Toxicologic evaluation of acute and subacute oral administration of Cucurbita maxima seed extracts to rats and swine. J. Ethnopharmacol., 43:45-51.

33. Winkler, C., G. Neurauter, K. Schroecksnadel, B. Wirleitner and D. Fuchs, 2005. Immunomodulatory Effects of Plant Extracts. In: Drug Development from New Molecules-Recent Progress in Medicinal Plants, vol. 11, Govil J.N., ed., Studium Press LLC, Houston, TX, 2005 (in press).
34. Wirleitner, B., K. Schroecksnadel, C. Winkler, H. Schennach and D. Fuchs, 2005. Resveratrol suppresses interferon-gamma-induced biochemical pathways in human peripheral blood mononuclear cells in vitro. Immunol. Lett. (in press).

35. Hung, H.C., K.J. Joshipura, R. Jiang, F.B. Hu, D. Hunter, S.A. Smith-Warner, G.A. Colditz, B. Rosner, D. Spiegelman and W.C. Willett, 2004. Fruit and vegetable intake and risk of major chronic disease. J. Natl. Cancer Inst., 96: 1577-84. 